\title{
Social Media für die Pferdepraxis
}

\author{
Nicole Biermann-Wehmeyer
}

Social Media Angebote werden von vielen Unternehmern, Ärzten und Tierärzten immer noch belächelt. Facebook? Unseriös und wenig hilfreich. Natürlich kann man diese Bedenken und Zweifel nachvollziehen: Als Tierarzt ist die Seriosität extrem wichtig. Man möchte schließlich eine Vertrauensperson für die Tierhalter darstellen. Hinzu kommen die rechtlichen Auflagen des unlauteren Wettbewerbsgesetzes und des Heilmittelwerbegesetzes. Es darf nicht gegen die ärztliche Schweigepflicht oder das Fernbehandlungsverbot verstoßen werden. Aber kann man sich den sozialen Netzwerken überhaupt entziehen?

\section{Hintergründe}

Wichtig ist, dass man die Zusammenhänge und Hintergründe der Social-MediaPortale versteht. In den 90er Jahren begann die kommerzielle Nutzung des Internets und es gehörte lediglich zum guten Ton, sich als Tierarztpraxis mit Portfolio, Leistungsspektrum, Öffnungszeiten und den wichtigsten Daten im Netz zu präsentieren.

Ab 2005 spricht man vom Web 2.0: Symptomatisch für diesen Begriff und den übergeordneten Social-Media-Gedanken ist der gegenseitige Austausch im Netz. Dieser Austausch findet zunehmend über das Handy statt. Prognosen gehen davon aus, dass im Jahr 2020 die wichtigste Verbindung zum Internet das Handy sein wird. Google wird ab Mitte April 2015 auf die Darstellung der Website beim Aufrufen der Seite über ein Handy achten.

Benutzerfreundliche Seiten mit anpassenden Darstellungen der Seite an den Handy- oder Tablet-Bildschirm werden dem Suchenden vorrangig vorgeschlagen.
Die sozialen Netzwerke Facebook, Twitter, Pinterest, YouTube und Co. bedienen den Wunsch der Kunden nach Portalen mit Mitmachcharakter. Sie sind geprägt durch das Kommentieren, Liken, Posten und Teilen. Es regiert die aktive Teilnahme der User und die Interaktion sollte möglichst authentisch und zeitnah erfolgen.

\section{Die Praxis-Homepage}

Für Tierärzte sollte die moderne Ausrichtung gegebenenfalls mit der Überarbeitung der eigenen Homepage starten.

\section{- pS}

Eine Internetpräsenz, die nur über statischen Content verfügt, ist nicht mehr zeitgemäß. Die Inhalte sollten regelmäßig überarbeitet werden.

Ideal ist die Kommunikation über einen Praxis-Blog. Dort können alle aktuellen Neuigkeiten zu der Praxis und den besonderen Dienstleistungen veröffentlicht werden. In einem Blog steht der neueste Beitrag aufgrund der Aktualität ganz oben. Weiterhin sollten die Interessenten die Beiträge kommentieren können. Dialog und Content sind King.

Der Blog sollte nicht nur verbale Aussagen enthalten, sondern auch multimediale Formate berücksichtigen: Fotos, Videos und Sprachaufzeichnungen. Das Einstellen solcher Beiträge regt selbstverständlich auch die Suchmaschinenoptimierung an. Der Marktführer Google versucht, dem Nutzer die bestmöglichen Beiträge im Netz zu dem gesuchten Schlagwort zu präsentieren. Diese sind i.d.R. multimedial, da sie viele Detailinformationen liefern. Einige neue Tierarztpraxen sind darauf angewiesen, dass sie im Netz gut gefunden werden. Dafür sind die genannten Bemühungen entscheidend.
Wenn die eigene Website den modernen Richtlinien entspricht, ist eine Anbindung an die Social Media Kanäle eine sinnvolle Ergänzung. Backlinks auf die eigene Homepage regen dabei die Suchmaschine zusätzlich an ( $\bullet$ Abb. 1).

\section{Facebook als Marketing-Instrument}

Die Anbindung an die sozialen Netzwerke beginnt in vielen Fällen mit Facebook.

\section{Die Facebook Fanpage}

Zunächst ist die Einrichtung einer Facebook Fanpage mit Impressum und den sonstigen rechtlichen Voraussetzungen ratsam. Die Gestaltung dieser Seite ist auch ohne Mediengestalter möglich, da sie von der Aktualität und Spontaneität lebt. Professionelle Fotos tragen natürlich dazu bei, dass die Produkte oder Dienstleistungen sehr positiv dargestellt werden. Auf Facebook ist es aber wichtig, dass die Fans der Seite merken, dass die Unternehmer authentisch agieren.

Die Inhalte der Facebook Fanpage können ganz unterschiedlich sein, z. B.:

- besondere Behandlungsmethoden

- Fotos von Tieren mit diversen Symptomen

- Erste-Hilfe-Tipps

- Serviceinformationen

Viele wissen gar nicht, dass man auch auf fremde YouTube-Videos verlinken kann und diese posten darf. Um die entsprechenden Inhalte im Netz zu finden, wäre es empfehlenswert, einen Google Alert einzustellen. Es wird damit möglich, aktuelle Inhalte im Netz zu verfolgen und die Themengebiete herauszufiltern, die Sie für Ihre Patienten und Tierhalter in den Vordergrund stellen möchten. 


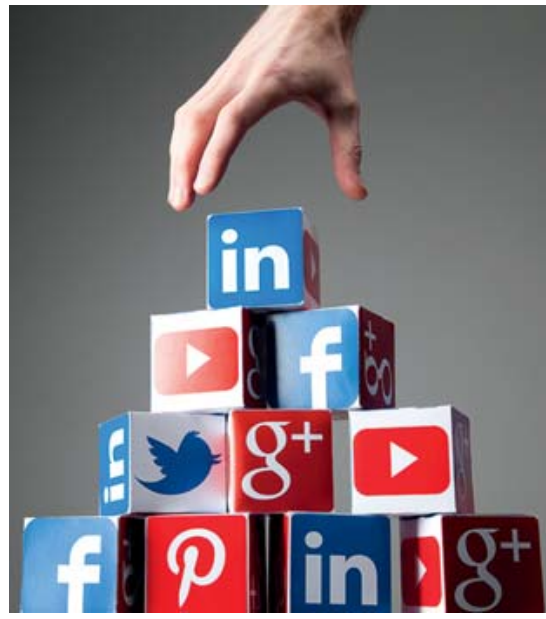

Abb. 1 Es ist sinnvoll, die Praxis-Webseite an die Social Media Portale anzubinden. (๑) mattjeacock, iStockphoto

Eine weitere Möglichkeit ist es, auf konkrete Gefahren hinzuweisen oder Empfehlungen bzgl. diverser Präventionsmethoden zu geben. Selbstverständlich ist es auch sinnvoll, mal eine positive oder lustige Geschichte zu erzählen. Da gibt es ja immer etwas Schönes zu berichten.

Bei Facebook ist es außerdem möglich, Veranstaltungen einzustellen und diese mit einer Gästeliste zu versehen. Wenn Sie merken, dass viele Patienten ein bestimmtes Thema interessiert, kann man durch eine solche Informationsveranstaltung sicherlich Vertrauen schaffen und aufklären. Eine dauerhafte Patientenbindung kann sich entwickeln. Die Veranstaltungen kann man außerdem über eine exakte Zielgruppeneinstellung wunderbar bewerben. Die Teilnahme der Angesprochenen wird außerdem der Freundesgruppe dieser Person angezeigt, sodass sehr häufig der gewünschte Schneeballeffekt erreicht wird.

\section{Werbeanzeigen}

Facebook steht häufig in der Kritik, weil so viele private Daten der User generiert werden. Das ist nicht grundsätzlich nachteilig für eine Tierarztpraxis. Im Gegenteil. Gerade bei überschaubaren Budgets für das Marketing kann Facebook sehr wertvoll sein. Man kann ein Angebot, eine Dienstleistung, einen bestimmten Beitrag oder eine Veranstaltung für eine speziell festgelegte Zielgruppe bewerben. Das ist beinahe ohne Streuverluste möglich, da
Facebook ganz genau weiß, welche Vorlieben der jeweilige Nutzer des Profils hat. Das Alter, das Geschlecht und die Interessen, z.B. „Pferde“, können spezifiziert werden. Der Beitrag, für den Sie eine „Werbeanzeige“ bei Facebook schalten, wird dann den Facebook-Nutzern innerhalb der Zielgruppe, die Sie festgelegt haben, in die private Startseite oder den Newsfeed eingespielt. Die Werbeanzeigen werden durch Facebook ausgewertet und ermöglichen ein lückenloses Monitoring durch die Unternehmer.

\section{Twitter}

Twitter wird meistens zur Verbreitung von Links genutzt, da man in diesem Portal bei einer öffentlich gemachten Nachricht (Tweet) nur 140 Zeichen zur Verfügung hat. Haben Sie einen interessanten Beitrag in einem Blog zu einer bestimmten Behandlungsmethode einer Pferdekrankheit geschrieben, können Sie diesen über Twitter in einem Tweet an Ihre Follower verbreiten.

Ein Tweet ist also eine Nachricht, die öffentlich erscheint. Insbesondere die Leute, die die Nachrichten eines Accounts abonniert haben (Follower), bekommen die Möglichkeit der Kenntnisnahme.

Selbstverständlich können Sie auch anderen Accounts und Persönlichkeiten, Kliniken oder Praxen auf Twitter folgen. Es ist keine Gegenseitigkeit erforderlich.

\section{YouTube}

Sehr wichtig ist auch das Portal YouTube. Eine kurze Anleitung mit visueller Demonstration, wie man z.B. eine Salbe bei Pferden an bestimmten Stellen aufträgt, kann zu großer Zeitersparnis führen. Schicken Sie den Tierhaltern einfach den Link zu Ihrem Demonstrationsvideo auf YouTube, selbstverständlich nur an die Tierhalter, die damit etwas anfangen können und das als Service und nicht als Beleidigung empfinden. Viele Menschen können auch nach eingehender telefonischer Beratung und Anleitung die Methoden nicht umsetzen, aber einem Video kann man in der Regel sehr gut folgen. Man kann es zudem wiederholen und zwischendurch anhalten.
Beim Hochladen der Videos können Sie eine Überschrift einsetzen, das Video beschreiben und einen Tag (Verschlagwortung) generieren. Das ist sehr sinnvoll, da YouTube die zweitgrößte Suchmaschine der Welt ist.

\section{Google+}

Mit Google+ wurde 2011 ein weiteres soziales Netzwerk eröffnet. Wie bei Facebook sind persönliche Profilseiten und Unternehmensseiten möglich und Veranstaltungen können publiziert werden. Der „+1“-Button ist vergleichbar mit dem „Like“-Button bei Facebook.

Das Portal Google+ bietet Unterstützung für die Informationsverbreitung und Suchmaschinenoptimierung. Im Vergleich zu Facebook hat Google+ eine geringere Verweildauer der User vorzuweisen. Bei der Verwendung von Google-Diensten wird automatisch ein Google+ Konto eröffnet

Google ist die am häufigsten verwendete Suchmaschine im deutschsprachigen Raum, daher kann man über eine Platzierung in Form einer Unternehmensseite/ Praxisseite auf Google+ nachdenken.

\section{Social Media Portale im medizinischen Bereich}

Jameda ist ein deutsches Arztbewertungsportal mit der Möglichkeit, sich als kompetenter Ansprechpartner zu qualifizieren. Tierhalter können Tierärzte bewerten und man kann sich anschauen, was andere Experten von sich geben. Zunehmend lassen sich Tierhalter von solchen Portalen beeinflussen.

Sehr zu empfehlen ist NOVICE (Network Of Veterinarians in Continuing Education, http://www.noviceproject.eu). Es handelt sich dabei um ein soziales professionelles online-Netzwerk für Tiermediziner, Dozierende und Studierende der Tiermedizin sowie für E-Learning-Berater bzw. Didaktiker der veterinärmedizinischen Bildungsstätten. Das Netzwerk soll die Zusammenarbeit der Bildungsstätten unterstützen. Ziel des Projektes ist die Förderung der Integration von Web 2.0-Werkzeugen zur Aus-, Fort- und Weiterbildung. 


\section{Andere Portale}

Die Bildportale Pinterest, FlickR und Instagram sind schön anzusehen, haben aber aus Sicht der Autorin keine sehr hohe Relevanz im tiermedizinischen Sektor. Wichtig ist dagegen ein vernünftiger Eintrag bei Google Places/Maps, der eine Art Visitenkarte darstellt.

Die mobile App Foursquare bekommt immer mehr Nutzer, da es sehr praktisch ist, sich mobil den Standort der Praxis anzusehen. Ein Eintrag ist kostenfrei und schadet Ihrer Praxis sicherlich nicht.

Ein wichtiger Faktor bei allen Social Media Portalen ist das Social Recruiting. Der Wettbewerb um die besten Fachkräfte oder Kooperationspartner findet auch für Tierarztpraxen in den sozialen Medien statt. Xing und Linkedin sind BusinessNetzwerke, aber auch über Facebook und YouTube werben große Praxen für eine gute Reputation als Ausbildungspraxis und Sympathieträger. Es können z. B. YouTube-Videos eingestellt werden, die Mitarbeiter bei täglichen Arbeiten zeigen und die Attraktivität des Unternehmens als Arbeitgeber unterstreichen. Die jungen Leute werden dort abgeholt, wo sie sich aufhalten: bei Facebook, YouTube und Co.

\section{.ps}

Es ist sinnvoll, in allen Portalen den gleichen Namen zu benutzen, der kurz und einprägsam ist.

Dieser Name sollte fortführend benutzt werden, da sich viele User in mehreren Portalen bewegen. Dies stärkt den Wiedererkennungswert.

\section{Tipps aus juristischer Sicht}

\section{Patientenbezogene Daten}

Mit patientenbezogenen Daten sollte man vorsichtig sein. Holen Sie sich im besten Fall eine vorherige Einwilligung der Tierhalter zur „Veröffentlichung ohne konkrete Namen“ ein, denn auch die Anonymi- sierung reicht oft nicht aus, wenn sich die User die fehlenden Informationen zur Identitätsfeststellung leicht beschaffen können und der Betroffene wider Willen ausfindig gemacht werden kann. Das Brechen der Schweigepflicht hat unter Umständen strafrechtliche Konsequenzen ( $\$ 203$ STGB). Auch zivilrechtliche und berufsrechtliche Probleme sind vorprogrammiert.

\section{Negative Äußerungen}

In Deutschland gibt es die Möglichkeit der freien Meinungsäußerung, die gerade aufgrund unserer Historie sehr ernst genommen wird. Bitte achten Sie darauf, dass Sie keine Äußerungen mit diffamierendem Inhalt posten. Solche Äußerungen über Personen, Personengruppen, Kollegen oder Tierhalter sollte man unterlassen. Wenn es wirklich sein muss, deklarieren Sie die Aussage als reine Meinungsäußerung und bleiben Sie sachlich.

\section{Werbung}

Beachten Sie das Transparenzgebot: Kommerzielle Werbung muss erkennbar sein (keine Verschleierung und kein Verstoß gegen das Gesetz gegen den unlauteren Wettbewerb oder das Telemediengesetz). Dazu kommt das Heilmittelwerbegesetz: Die Werbung muss wahr sein ( $\$ 3$ HWG), suggestive Werbemethoden sind im Gesundheitsbereich unerwünscht. Werbung mit ärztlichen oder sonstigen fachlichen Empfehlungen ist unzulässig (§11 HWG).

\section{Weitere Tipps}

Das Tierarzt-Tierhalter-Verhältnis sollte nicht in ein Tierarzt-Online-FreundschaftVerhältnis verwandelt werden. Wenn Sie privat online sind, müssen Sie sich nicht genötigt fühlen, über Facebook noch bis tief in die Nacht Fragen zu beantworten. Kurze Antworten zu angemessenen Zeiten sind in Ordnung, aber Sie sollten das festlegen. Die Kunden sollten vorwiegend in die Praxis kommen, um auch eine vernünftige Abrechnung der Leistungen möglich zu machen. Ziehen Sie sich in diesen Fällen auf das Fernbehandlungsverbot zurück. Danach dürfen Beratungen nur in
Grenzen über Print- und Kommunikationsmedien durchgeführt werden. Auch in der Telemedizin herrscht der Grundsatz der Unmittelbarkeit der Behandlung vor. In diesem Zusammenhang wird sich in Zukunft sicherlich noch einiges wandeln und öffnen.

\section{Fazit}

Die Auftritte der Tierarztpraxen in den sozialen Medien sind nicht mehr wegzudenken. Ob man diese Trends nun gut oder schlecht findet, jeder Tierarzt sollte sich über eine Platzierung in den neuen Medien grundsätzlich Gedanken machen. Eine moderne Darstellung der Tierarztpraxis ist sehr wichtig und wenn man zu einem späteren Zeitpunkt die Praxis verkaufen möchte, spielt die bereits aufgebaute Internetpräsenz eine immer wichtigere Rolle.

Die Pflege der Portale ist natürlich zeitaufwendig, aber man erreicht nach dem Aufbau sehr viele Tierhalter gezielt. Eine externe Beratung und Vergabe ist auch möglich und vielleicht sinnvoll, der Auftritt muss aber in jedem Fall authentisch bleiben.

Auch wenn die Tierarztpraxis momentan sehr gut ausgelastet ist, sollte ein Social Media Konzept in die Strategieausrichtung und Vision für die Zukunft mit einbezogen werden. Ausgangspunkt sollte selbstverständlich immer die Website der Praxis sein. Diese ist Ihr Eigentum und Ihr Inhalt, die Seiten in den Portalen nicht.

\section{Online}

http://dx.doi.org/10.1055/s-0035-1545971

Nicole Biermann-Wehmeyer
Juristin und Dozentin
BIW - Bildungsinstitut Wirtschaft
Seminare für Existenzgründung, Buchführung,
Social Media Marketing und Rhetorik
Up de Welle 17
46399 Bocholt
info@bildungsinstitut-wirtschaft.de

